\title{
Electron Bunch Timing with Femtosecond Precision in a Superconducting Free-Electron Laser
}

\author{
F. Löhl, ${ }^{1, *}$ V. Arsov, ${ }^{1, \dagger}$ M. Felber, ${ }^{1}$ K. Hacker, ${ }^{1}$ W. Jalmuzna, ${ }^{2}$ B. Lorbeer, ${ }^{1}$ F. Ludwig, ${ }^{1}$ K.-H. Matthiesen, ${ }^{1}$ H. Schlarb, ${ }^{1}$ \\ B. Schmidt, ${ }^{1}$ P. Schmüser, ${ }^{3}$ S. Schulz,${ }^{3}$ J. Szewinski, ${ }^{4}$ A. Winter, ${ }^{1, \$}$ and J. Zemella ${ }^{3}$ \\ ${ }^{1}$ Deutsches Elektronen-Synchrotron DESY, Notkestrasse 85, 22607 Hamburg, Germany \\ ${ }^{2}$ Technical University of Lodz, al. Politechniki 11, 93-590 Lodz, Poland \\ ${ }^{3}$ University of Hamburg, Institute of Experimental Physics, Luruper Chaussee 149, 22761 Hamburg, Germany \\ ${ }^{4}$ The Andrzej Soltan Institute for Nuclear Studies, Otwock-Swierk 05-400, Poland
}

(Received 17 January 2010; published 5 April 2010)

\begin{abstract}
High-gain free-electron lasers (FELs) are capable of generating femtosecond x-ray pulses with peak brilliances many orders of magnitude higher than at other existing X-ray sources. In order to fully exploit the opportunities offered by these femtosecond light pulses in time-resolved experiments, an unprecedented synchronization accuracy is required. In this Letter, we distributed the pulse train of a mode-locked fiber laser with femtosecond stability to different locations in the linear accelerator of the soft x-ray FEL FLASH. A novel electro-optic detection scheme was applied to measure the electron bunch arrival time with an as yet unrivaled precision of $6 \mathrm{fs}$ (rms). With two beam-based feedback systems we succeeded in stabilizing both the arrival time and the electron bunch compression process within two magnetic chicanes, yielding a significant reduction of the FEL pulse energy jitter.
\end{abstract}

DOI: 10.1103/PhysRevLett.104.144801

PACS numbers: 41.60.Cr, 29.20.Ej, 41.75.Ht, 42.65.Re

High brightness $\mathrm{x}$-ray pulses of femtosecond duration have been a dream in many disciplines of science. The short wavelength provides atomic spatial resolution and permits the study of the structure and properties of single atoms, large macromolecules, and biological systems. High-gain free-electron lasers are capable of generating such an x-ray beam and in contrast to conventional synchrotron light sources, the x-ray pulse duration is in the femtosecond rather than in the nanosecond or picosecond range. This allows for resolving the evolution of physical and chemical processes on the femtosecond scale [1]. The up to $10^{12}$ photons per single $\mathrm{x}$-ray pulse allow for capturing complete diffraction images on a single shot basis [2] and in combination with the ultrashort pulse durations very high peak intensities are reached, permitting the research on strongly nonlinear interactions.

At the ultraviolet and soft $\mathrm{x}$-ray free-electron laser (FEL), FLASH at DESY, Hamburg, an FEL pulse duration of 8 fs was determined at a wavelength of $13 \mathrm{~nm}$ [3]. Experimental studies at the Linac Coherent Light Source (LCLS) in Stanford, California, the first FEL operating in the hard $\mathrm{x}$-ray regime, indicate the feasibility of generating light pulses with durations of only a few femtoseconds [4]. With special electron beam manipulation techniques, in which a fraction of the driving electron bunch is energy modulated by a few-cycle laser pulse in a short undulator segment, even the generation of attosecond pulses is envisioned (see, e.g., [5-7]).

The major challenge associated with such ultrashort light pulses is a correspondingly precise synchronization of the linear accelerator (linac) driving the FEL process. Conventional radio frequency (rf) synchronization methods have not yet demonstrated long term synchronization with femtosecond stability over large distances. The most promising alternative is a laser-optical synchronization system in which the periodic pulse train of a near-infrared fiber laser is distributed in the accelerator tunnel via lengthstabilized optical fibers. Passively mode-locked lasers feature a very high intrinsic timing stability (see, e.g., [8]), and with suitable corrections of the laser resonator length even subfemtosecond stability is achievable [9]. In order to demonstrate the viability of the laser-based optical synchronization system under construction for FLASH, we implemented two laser-driven beam arrival-time monitors in the linac tunnel to carry out measurements on the time resolution that can be achieved with a laser-based synchronization system.

The timing of the FEL light pulses is determined by the arrival times of the driving electron bunches at the undulator. The self-amplified spontaneous emission (SASE) FEL process leads to additional statistical fluctuations on the order of the cooperation time, which is a few femtoseconds for soft X-ray FELs and much less than 1 fs for hard x-ray FELs. Electron beam timing jitter is introduced at various locations along the accelerator. The main contribution is due to dispersive effects in the magnetic bunch compressor chicanes, which generate the high local charge density needed in the high-gain FEL process. The electron bunches are accelerated off-crest in the accelerating cavities preceding the magnetic chicanes in order to imprint an energy gradient along the bunch. The energy-dependent path length in a magnetic chicane leads to the desired longitudinal compression, but at the same time it converts any beam energy jitter into an arrival-time jitter at the undulator. Beam energy jitter at the chicane location can either be caused by amplitude fluctuations of the acceler- 


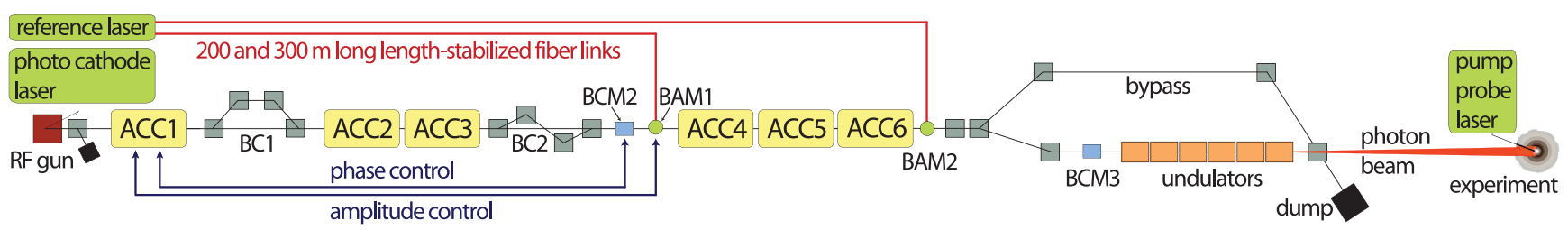

FIG. 1 (color online). Schematic of the free-electron laser FLASH with the locations of the two bunch arrival-time monitors (BAM1, BAM2) and the two bunch compression monitors (BCM2, BCM3). ACC1-ACC6: accelerating modules, BC1 and BC2: bunch compressors.

ating fields in the cavities upstream of the chicane or by variations of the beam phase, i.e., the phase of the rf field in the cavity with respect to the arrival-time of the bunch. A beam phase jitter can be induced by either phase jitter of the accelerating fields or by time jitter of the electron gun. In the latter case, the resulting energy deviations lead to time-of-flight variations in the chicane which counteract the initial arrival-time fluctuations and reduce them by the bunch compression factor (the ratio of bunch lengths upstream and downstream of the chicane). At FLASH, the beam arrival-time jitter at the undulators is dominated by amplitude fluctuations of the accelerating fields in the first accelerating module ACC1 and the resulting time-of-flight variations due to the first bunch compressor $\mathrm{BC} 1$ (see Fig. 1).

An important component of the beam arrival-time monitor (BAM) is an erbium-doped soliton fiber laser providing a laser pulse train with a precisely controlled repetition rate of $216.7 \mathrm{MHz}$, the sixth subharmonic of the $1300 \mathrm{MHz}$ radio frequency. The sub-100 fs long infrared pulses are distributed along the accelerator using dispersion compensated optical fiber links. The optical length of each link is stabilized using a piezoelectric fiber stretcher. At the end of the link, the laser intensity is partly reflected, and the reflected pulse train travels back through the fiber to be cross correlated with the incoming laser pulse train. The sum-frequency signal of the optical cross correlator drives a feedback loop acting on the fiber-stretcher to correct fiber length changes with femtosecond precision [10].

The key component of the BAM is a novel electro-optic detection scheme. The beam-induced bipolar signal from a
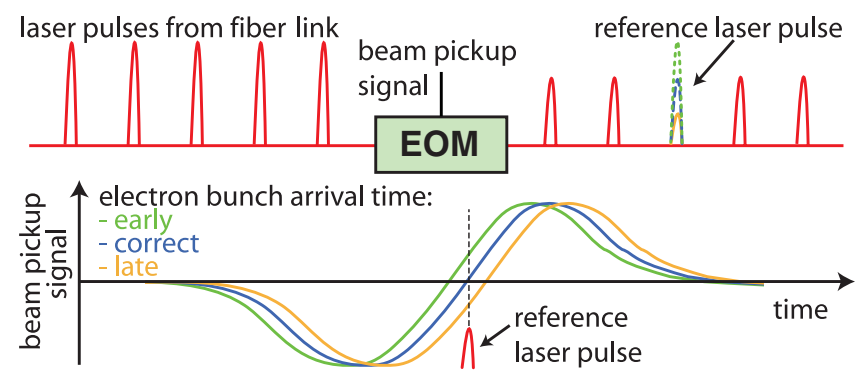

FIG. 2 (color online). Operation principle of the electron bunch arrival-time measurement using an electro-optic modulator $(\mathrm{EOM})$ pickup electrode with more than $10 \mathrm{GHz}$ bandwidth is utilized to modulate the amplitude of the laser pulse train by means of a commercial Mach-Zehnder type electrooptic modulator (EOM). The principle of the arrival-time monitor is explained in Fig. 2. The relative timing is adjusted such that one laser pulse out of the train samples the zero crossing of the pickup signal. A small shift in the electron bunch timing moves the zero-crossing position so that the laser pulse experiences a modulation voltage. By comparing the pulse energy of the sampling pulse to that of the adjacent laser pulses, one can deduce the electron bunch arrival time with high accuracy.

Two bunch arrival-time monitors, BAM1 and BAM2, were installed in a $60 \mathrm{~m}$ long straight section of the accelerator. Each monitor was supplied with laser pulses through its own stabilized fiber link having lengths of 200 and $300 \mathrm{~m}$, respectively. Figure 3 (top) shows the arrival times of 1500 bunches as measured with the two detectors. The arrival-time variation is about $200 \mathrm{fs}$ ( $\mathrm{rms}$ ) with a strong correlation between BAM1 and BAM2. The bottom plot of Fig. 3 shows that the difference signal of both detectors has an rms variation of only $8.5 \mathrm{fs}$. Thus the BAM resolution can be estimated to be $8.5 \mathrm{fs} / \sqrt{2}=6 \mathrm{fs}$. This is a factor of 10 improvement compared to previous
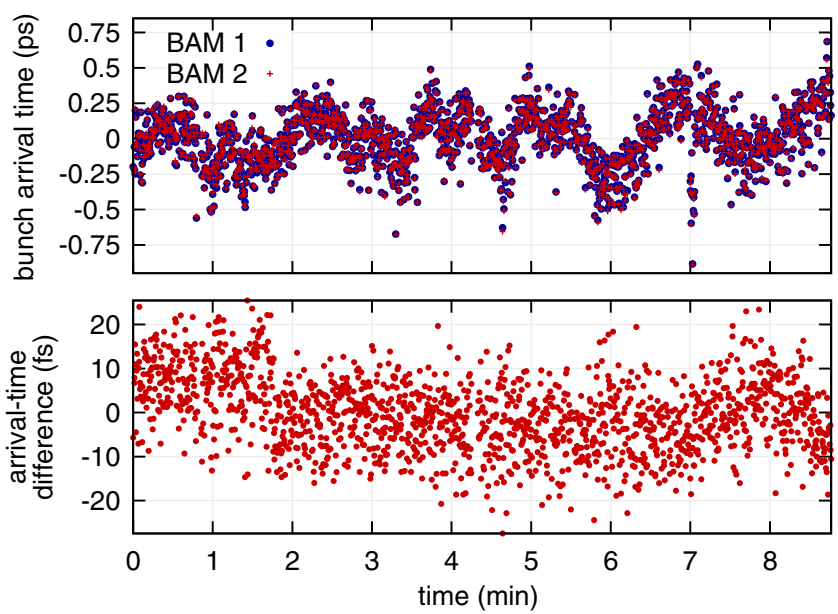

FIG. 3 (color online). Top: Measured arrival times of 1500 consecutive electron bunches at two locations (BAM1 and BAM2) in the linac. The bunch charge is $0.8 \mathrm{nC}$. Bottom: Difference signal of the two BAMs. 


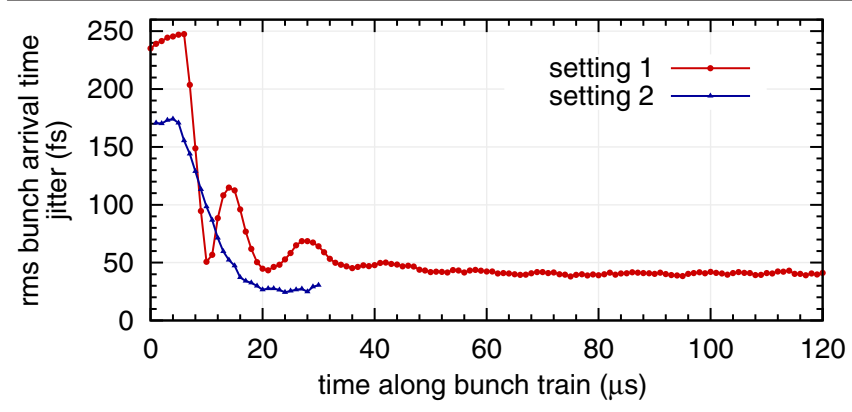

FIG. 4 (color online). Effect of the bunch arrival-time feedback on the arrival-time jitter along bunch train for two different machine settings. A stability of 25 fs could be achieved in the best case (setting 2).

electro-optic measurements [11]. Over longer time durations, the estimated BAM resolution is slightly worse with around 9 fs over $1.5 \mathrm{~h}$ and $14 \mathrm{fs}$ over $4.5 \mathrm{~h}$. Since we observed steps in the difference signal of both BAMs, we believe that this is caused by changes in the electron bunch shape due to machine tuning, and a slightly different response characteristics of the beam pickups used in both BAMs. This inherent interdependence of the BAM on the longitudinal shape of the bunch will be greatly attenuated by the foreseen linearized bunch compression scheme (also used at LCLS) which removes the picosecond long tails in the charge distribution.

The signal from the arrival-time monitor BAM1 was utilized to establish an arrival-time feedback acting on the rf field amplitude $A$ of the first accelerating module. This possibility is unique to superconducting accelerators due to the long duration of the rf pulse (800 $\mu$ s at FLASH). Figure 4 shows the effect of the feedback on the arrivaltime jitter along the bunch train. The results for two different accelerator settings are shown. In a first measurement (setting 1), the timing jitter without feedback was $240 \mathrm{fs}$, visible at the first few bunches in the train, which are not stabilized due to the feedback latency of about $6 \mu \mathrm{s}$. The arrival-time fluctuation of the later bunches is reduced to $40 \mathrm{fs}$. This number is far larger than the BAM resolution and dominated by fast fluctuations occurring within the feedback latency time. The second curve (setting 2) shows the stabilization process for a case in which fast arrivaltime fluctuations from the photocathode laser have been reduced, and the latency of the feedback has been decreased. These two improvements reduced the arrivaltime fluctuation to $180 \mathrm{fs}$ without and $25 \mathrm{fs}$ with the feedback. Only short bunch trains were possible at this time due to technical reasons. Note that the unstabilized first part of the bunch train can in principle be prevented from generating FEL radiation by disturbing the beam orbit in the undulator with a fast kicker magnet. The arrival-time feedback permits the generation of predefined arrival-time distributions along the bunch train. Figure 5 shows some examples. In pump-probe experiments, this may allow for a complete delay scan within a single bunch train.

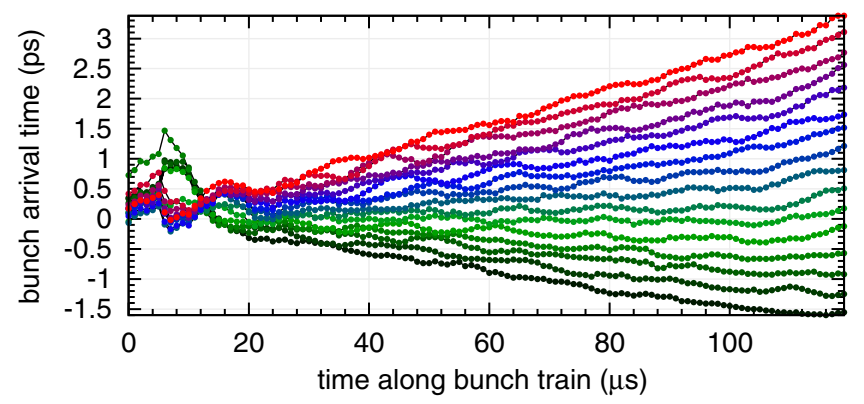

FIG. 5 (color online). Generation of different arrival-time distributions along the electron bunch train using the bunch arrivaltime feedback. Each color indicates a predefined arrival-time slope within a single bunch train.

It should be noted that the beam arrival-time stabilization we describe differs from an energy feedback based on beam position measurements inside of a bunch compressor chicane. Timing fluctuations of the gun occurring slower than the feedback bandwidth would not be reduced anymore in the bunch compressor chicanes if a pure energy feedback is deployed.

The bunch compression process in the magnetic chicanes depends strongly on the beam phase in the cavities of the first accelerating module ACC1. Beam phase fluctuations, caused either by $\mathrm{rf}$ phase fluctuations or by arrival-time jitter of the electron beam coming from the injector, lead to a stronger or weaker compression of the bunch which can be detected by monitoring the intensity of coherent radiation. A bunch compression monitor BCM2, consisting of a diffraction radiator and a pyroelectric detector, is installed behind the second bunch compressor $\mathrm{BC} 2$. The integrated diffraction radiation power measured with BCM2 was utilized in a second feedback loop to stabilize the beam phase in ACC1. The proper operation of this bunch compression feedback was verified with a second, independent bunch compression monitor, BCM3, located in front of the undulator magnets (see Fig. 1). The feedback reduced the bunch compression fluctuations by more than a factor of 5 (see Fig. 6). Since only the cavities of ACC1 were used to generate the required energy chirp

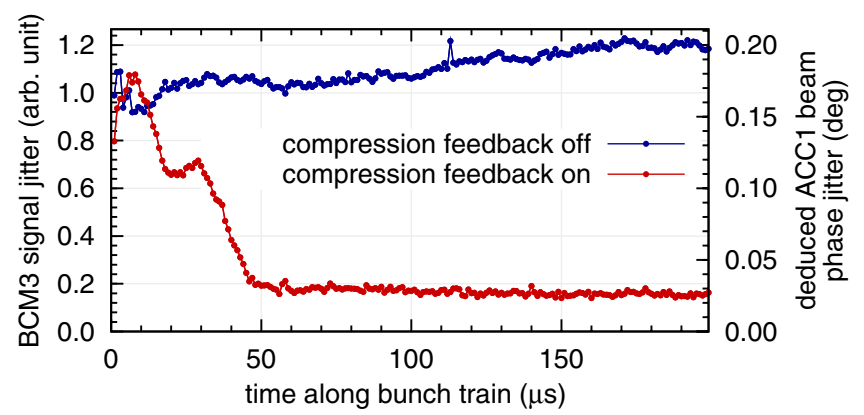

FIG. 6 (color online). Effect of the bunch compression feedback on the beam phase fluctuations along the bunch train as measured with the bunch compression monitor BCM3. 


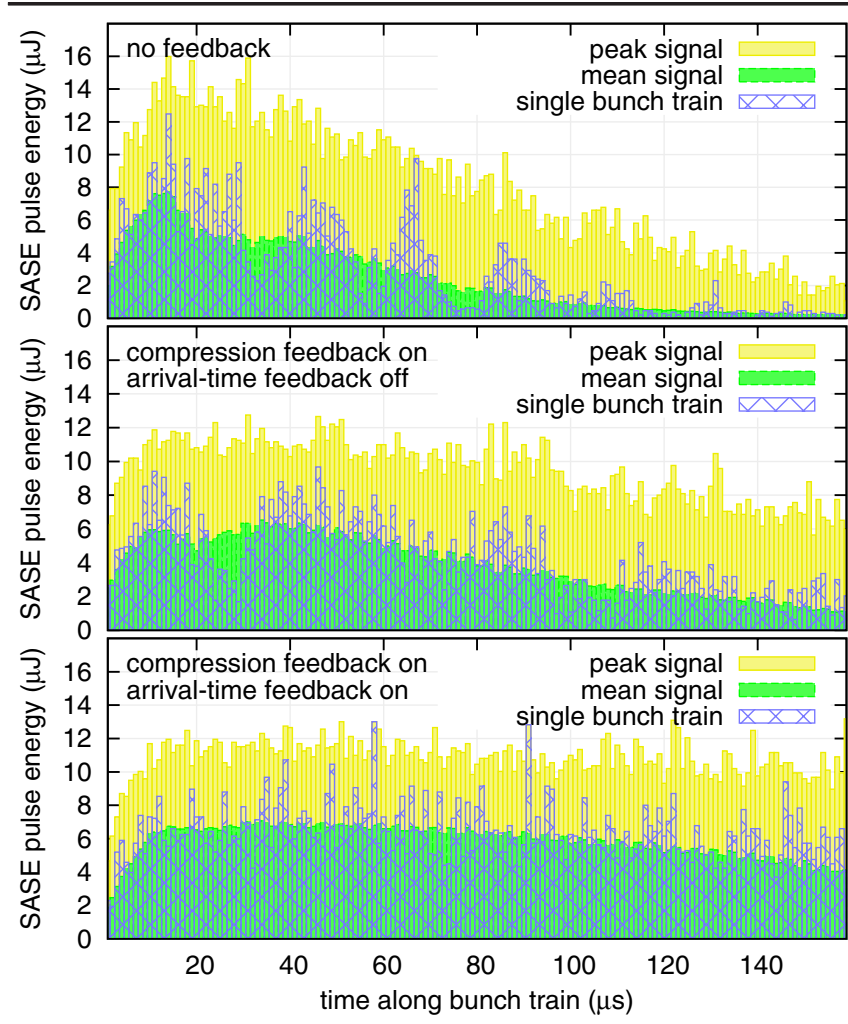

FIG. 7 (color online). Distribution of the FEL radiation pulse energy along the bunch train without and with activated bunch compression and arrival-time feedbacks.

along the bunch, we can deduce the beam phase stability in ACC1 from the bunch compression signal. It is improved from $0.2^{\circ}$ without to $0.025^{\circ}$ with the compression feedback.

Combining both feedback loops led to a significant improvement of the FEL pulse energy stability. Figure 7 shows the FEL pulse energy distribution along the bunch train for three different feedback configurations (no feedbacks, only bunch compression feedback, both feedbacks active). The peak and mean values are calculated over 600 consecutive bunch trains. Without any feedback, the FEL pulse energy decreases gradually along the bunch train. After activating both stabilization loops, the average pulse energy is more than doubled and remains nearly constant over the entire bunch train. Furthermore, the pulse energy fluctuations along the train are reduced significantly to around $20 \%$ as depicted in Fig. 8. The remaining fluctuations correspond to the statistical limit of the SASE process for the case in which the FEL is operated in the exponential growth regime and for 25 longitudinal modes present. This

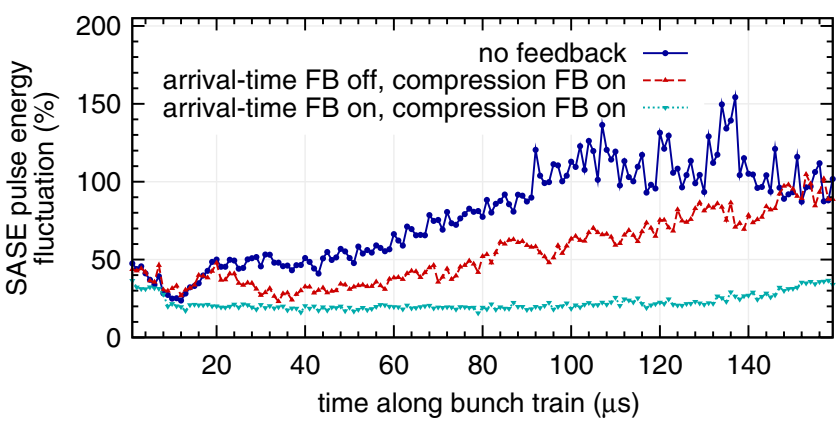

FIG. 8 (color online). Effect of the arrival-time feedback and the bunch compression feedback on the FEL pulse energy fluctuations along the bunch train.

is realistic, because the FEL performance was not optimized and not tuned for the shortest pulse lengths.

In conclusion, we demonstrated the viability of the laserbased optical synchronization of an FEL at the femtosecond level. Bunch arrival times were measured with a so far unrivaled precision of $6 \mathrm{fs}$ with respect to a remotely located reference laser. We were able to reduce the beam timing jitter to $25 \mathrm{fs}$. The combination of arrival-time and compression feedbacks improved the stability of the FEL pulse energy significantly. Time-resolved measurements with femtosecond precision will be possible by synchronizing the optical lasers, used in two color pump-probe experiments, to the reference fiber laser.

We would like to thank the FLASH team and our technicians for their support.

*florian.loehl@cornell.edu

Present address: Cornell University, Ithaca, NY, USA.

${ }^{\dagger}$ Present address: Paul Scherrer Institute, 5232 Villigen PSI, Switzerland.

"Present address: ITER Organization, 13067 St. Paul-lezDurance, France.

[1] Th. Tschentscher, Chem. Phys. 299, 271 (2004).

[2] R. Neutze et al., Nature (London) 406, 752 (2000).

[3] W. Ackermann et al., Nat. Photon. 1, 336 (2007).

[4] Y. Ding et al., SLAC Report No. SLAC-PUB-13525, 2009.

[5] E. L. Saldin, E. A. Schneidmiller, and M. V. Yurkov, Opt. Commun. 237, 153 (2004).

[6] A. A. Zholents and W. M. Fawley, Phys. Rev. Lett. 92, 224801 (2004).

[7] G. Stupakov, Phys. Rev. Lett. 102, 074801 (2009).

[8] J. Kim et al., Opt. Lett. 32, 3519 (2007).

[9] T. R. Schibli et al., Opt. Lett. 28, 947 (2003).

[10] J. Kim et al., Opt. Lett. 32, 1044 (2007).

[11] A. L. Cavalieri et al., Phys. Rev. Lett. 94, 114801 (2005). 